\title{
Akt1 in the cardiovascular system: friend or foe?
}

\author{
Brian T. O'Neill and E. Dale Abel
}

Division of Endocrinology, Metabolism and Diabetes and Program in Human Molecular Biology and Genetics, University of Utah School of Medicine, Salt Lake City, Utah, USA.

\begin{abstract}
Akt is an important signaling molecule that modulates many cellular processes such as cell growth, survival, and metabolism. Akt activation has been proposed as a potential strategy for increasing cardiomyocyte survival following ischemia. In mammalian cells, 3 distinct isoforms of Akt exist, but their precise roles in cardiovascular biology were previously unknown. Three separate studies published in this issue of the JCI now provide important new insight into the central role of Akt1 in the regulation of angiogenesis and the maladaptive or deleterious consequences of chronic unregulated Akt activation in the heart (see the related articles beginning on pages 2108, 2119, and 2128). Here we discuss the implications of these exciting new studies.
\end{abstract}

The serine/threonine kinase Akt (also known as protein kinase $\mathrm{B}[\mathrm{PKB}])$ plays a central role in the regulation of cellular growth, survival, and metabolism across many species. In mammalian cells, there are 3 distinct Akt isoforms (Akt1, -2, and -3; also known as $\mathrm{PKB} \alpha,-\beta$, and $-\gamma$ ), which are the products of distinct genes (1). Gene knockout studies have provided evidence that these isoforms may serve distinct roles with varying degrees of functional overlap. For example, germline deletion of Akt 1 in mice leads to a predominant phenotype of reduced size for multiple organs, which is more the result of reduced cell size than reduced cell numbers. In contrast, Akt2 $\mathrm{KO}$ mice do not exhibit striking reductions in cell size, but develop insulin resistance and diabetes due to impaired insulin signaling in the liver and skeletal muscle and impaired survival of pancreatic $\beta$ cells (2-4). Akt3 KO mice exhibit a predominantly neurological phenotype with reduced brain size (5). While mice with individual Akt isoform KOs are viable, evidence for functional overlap of these isoforms comes from studies in mice with combinatorial KOs. Animals with multiple gene deletions exhibit much more severe phenotypes (which are not compatible with long-term survival) than those animals with single gene deletions (6). Akt isoforms can be activated in response to various growth factors and hormones, includ-

Nonstandard abbreviations used: $\mathrm{I} / \mathrm{R}$, ischemia/ reperfusion; IRS, insulin receptor substrate; $\mathrm{MTOR}$, mammalian TOR; PKB, protein kinase B; S6K, S6 kinase; TOR, target of rapamycin.

Conflict of interest: The authors have declared that no conflict of interest exists.

Citation for this article: J. Clin. Invest. 115:2059-2064 (2005). doi:10.1172/JCI25900. ing insulin, IGF-1, VEGF, and $\beta$-adrenergic stimulation. In the case of insulin, the insulin receptor tyrosine kinase phosphorylates and activates insulin receptor substrate-1 (IRS-1) and IRS-2, which then activate PI3K. PI3K activation generates phosphoinositide-3,4,5 triphosphate, which mediates the phosphorylation and activation of Akt. Once activated, Akt phosphorylates various intracellular signaling intermediates that regulate a diverse array of cellular processes (reviewed in ref. 7) (Figure 1A).

\section{Akt activation has been proposed as a potential strategy for increasing cardiomyocyte survival following ischemia.}

In the cardiovascular system, Akt plays an important role in the regulation of cardiac hypertrophy, angiogenesis, and apoptosis (7). The observation that acute activation of Akt in cardiomyocytes in vivo and in vitro protects against apoptosis after ischemia/reperfusion (I/R) injury has raised hopes that Akt activation could represent an important therapeutic strategy for limiting myocardial injury following ischemia (8) (Figure 1A). Moreover, activation of Akt in mesenchymal stem cells may enhance cardiac function in heart failure models to a greater extent than that observed in nontransduced stem cells $(9,10)$. Although the results of transgenic overexpression studies suggest that Akt overexpression may produce a compensated state of cardiac hypertrophy, other work suggests that this might be deleterious over the long term (11-13). Two separate reports in this issue of the JCI, the first by Nagoshi et al. (14) and the second by Shiojima et al. (15), provide important new insights into the potential mechanisms by which Akt overexpression in the heart may become maladaptive.

Another unresolved issue in the cardiovascular system was the specific role of the 3 Akt isoforms. For example, while it was clear that Akt mediates many of the proangiogenic effects of secreted peptides such as VEGF, it remained unknown which specific isoforms were responsible for mediating these effects in vivo (7). Similarly, while an essential role for PI3K/Akt signaling in the regulation of physiological (e.g., exerciseinduced) hypertrophy is well established $(16,17)$, it was not previously known which specific Akt isoform(s) were responsible. In this issue of the JCI, the study by Ackah et al. (18) now provides very strong evidence that Akt 1 is the specific Akt isoform that mediates adaptive angiogenesis.

\section{Mechanisms responsible for maladaptation in the heart when Akt is chronically overexpressed}

How much Akt is too much? Two studies in this issue describe distinct ways in which chronic unregulated Akt signaling in cardiomyocytes in vivo ultimately becomes maladaptive $(14,15)$. Both of these studies used mouse models with transgenic overexpression of an activated (myristoylated) form of Akt1. Shiojima and colleagues (15) used a tetracycline-regulated system that increased myocardial Akt kinase activity by approximately 15 -fold, while Nagoshi and colleagues (14) used a transgenic mouse with constitutive Akt 1 activation in which Akt kinase activity was increased to at least the extent of the Shiojima et al. study. The model reported by Shiojima and colleagues developed severe cardiac dysfunction following long-term Akt activation (15), while cardiac dysfunction in mice reported by Nagoshi et al. was less severe (14). Two other independent transgenic lines of myocardial Akt1 overexpression were previously reported $(12,13)$. High-level overexpression (greater than 80-fold) of a constitutively 
A

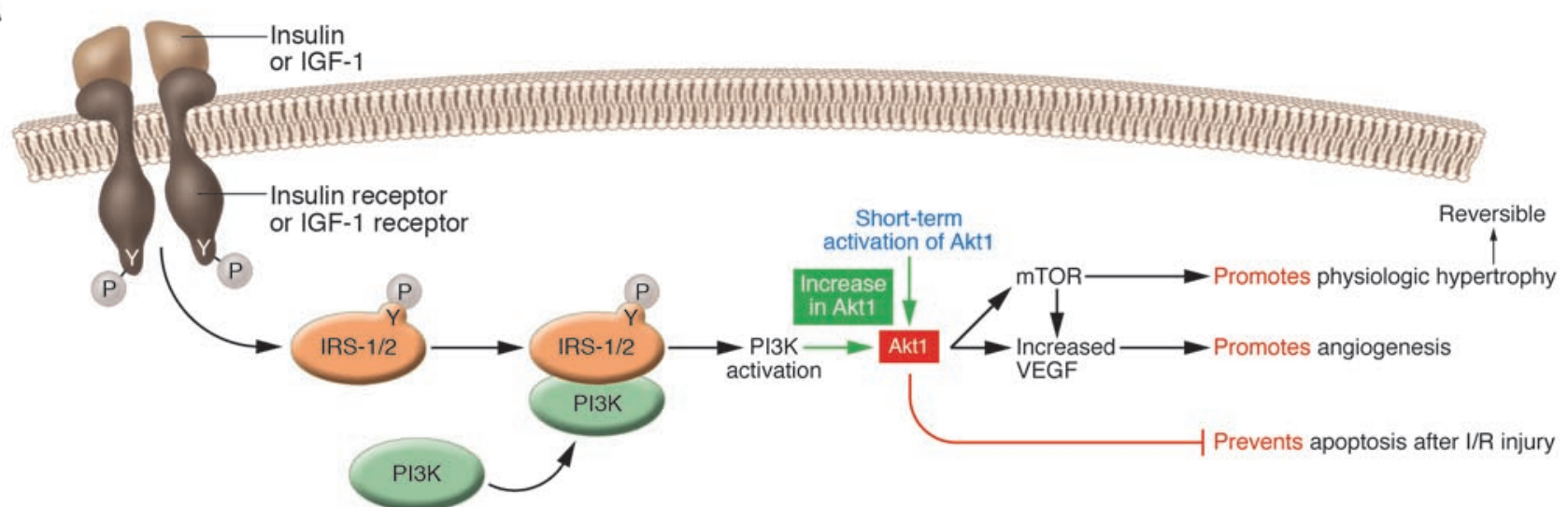

$\mathbf{B}$

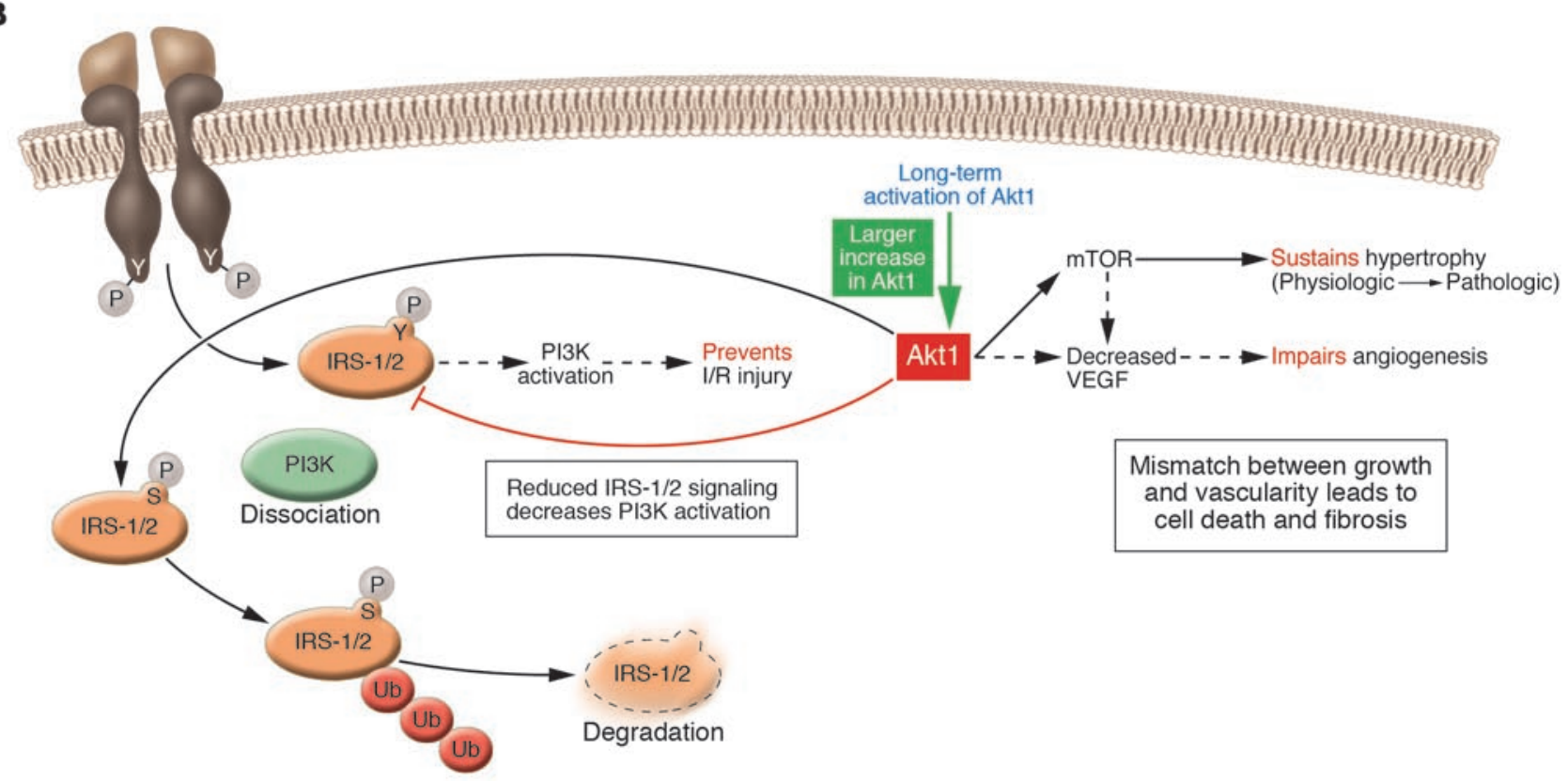

Figure 1

Effect of short-term or long-term Akt activation in cardiac muscle. (A) Short-term activation of Akt1 by physiological stimuli, such as insulin and IGF-1 signaling or exercise, promotes physiologic cardiac hypertrophy. Insulin and IGF-1 signaling is mediated in part via tyrosine phosphorylation (P) of IRS-1/2. Phosphorylated IRS-1/2 associates with and activates PI3K, which stimulates the generation of phosphoinositide-3,4,5 triphosphate and subsequently leads to the activation of Akt. Akt1 promotes physiologic hypertrophy via mTOR-mediated pathways. Shortterm activation is associated with increased VEGF generation and release and may also protect against I/R injury. (B) Long-term, high-level Akt signaling is maladaptive. Downregulation of IRS-1/2 proteins occurs via mechanisms that involve increased proteasomal degradation. It is believed that increased serine phosphorylation of IRS-1/2 will promote this degradation. In addition, serine phosphorylation of IRS-1/2 limits its association with the insulin receptor, which further reduces the activation of PI3K. Reduced PI3K activation is associated with reduced recovery and increased tissue injury following I/R due to loss of PI3K-dependent but Akt-independent cardioprotective mechanisms. It is also possible that reduced IRS-1-mediated PI3K signaling may contribute to cardiac injury in patients with dilated cardiomyopathy. Long-term activation of Akt continues to promote cardiac hypertrophy. However, there is downregulation of VEGF expression, which leads to reduced angiogenesis. A mismatch between cardiomyocyte growth and blood vessel growth leads to cell death and fibrosis. Ub, ubiquitination.

active Akt1 mutant, T308D/S473D, precipitated age-related LV dysfunction (13), whereas overexpression of another mutant Akt1, E40K, was associated with increased basal cardiac contractility but impaired inotropic response to dobutamine (12). Taken together, these models suggest that Akt 1 overexpression in the heart may pro- duce a broad phenotypic spectrum in the unstressed heart, but in all cases there was some evidence of cardiac maladaptation. In contrast, a PI3K transgenic mouse with 4-fold increase in the ratio of phosphorylated Akt/total Akt did not develop cardiac dysfunction (19). Moreover, in physiological contexts, Akt activation is increased 1.5- to 2-fold in response to exercise training (16) and 6-fold by transgenic overexpression of IGF-1 (20) or the IGF-1 receptor (17). In these situations cardiac dysfunction does not occur. Thus, interpretation of the phenotypes of transgenic models of Akt1 overexpression should always be tempered by the fact that the degree of 


\section{Table 1}

Divergence in long-term versus short-term effects of Akt signaling

\begin{tabular}{|c|c|c|c|c|c|c|}
\hline Akt activation & Hypertrophy & $\begin{array}{l}\text { Regression of } \\
\text { hypertrophy }\end{array}$ & $\begin{array}{c}\text { I/R } \\
\text { injury }\end{array}$ & Function & $\begin{array}{l}\text { Angiogenic } \\
\text { mediators }\end{array}$ & $\begin{array}{l}\text { IRS-1/PI3K } \\
\text { activation }\end{array}$ \\
\hline Short-term & Yes (15) & Yes (15) & Protected (14) & Normal (15) & Increased (15) & Unknown \\
\hline Long-term & Yes $(14,15)$ & No (15) & Not protected (14) & Decreased $(14,15)$ & Decreased (15) & Decreased (14) \\
\hline
\end{tabular}

References corresponding to these results are cited in parentheses.

transgene overexpression often far exceeds physiologically achieved levels.

The studies by Nagoshi et al. (14) and Shiojima et al. (15) provide mechanistic insights into the deleterious consequences of long-term Akt1 overexpression in the heart (Figure 1B and Table 1). Shiojima and colleagues (15) report that short-term (less than 2-week) activation of Akt1 in the

How much Akt is too
much? Two studies in this
issue describe distinct
ways in which chronic
unregulated Akt signaling
in cardiomyocytes in
vivo ultimately becomes
maladaptive.

heart leads to compensated cardiac hypertrophy that is completely reversible after transgene expression is turned off. In contrast, 6 weeks of transgene induction led to even greater LV hypertrophy and contractile dysfunction, both of which are associated with increased fibrosis and decreased capillary density. Thus, unlike short-term transgene activation in which VEGF expression is upregulated (in part by target of rapamycin-mediated [TOR-mediated] signaling) (Figure 1A), such adaptations are lost following longer-term activation of Akt (Figure 1B). This observation prompted Shiojima and colleagues to test the hypothesis that matched angiogenesis and cellular growth is required for maintenance of compensated hypertrophy following Akt activation. Blocking VEGF signaling - by systemic administration of an adenovirus that expressed the soluble VEGF receptor - reduced capillary density following short-term Akt activation, and this precipitated LV dysfunction and increased myocardial injury. This model raises the intriguing hypothesis that signaling cross-talk medi- ated by Akt 1 is required for the coordinate regulation of myocardial growth and angiogenesis during cardiac hypertrophy. As Akt is activated in failing hearts and in response to pressure overload, it will therefore be important to determine whether Akt-mediated VEGF release and subsequent angiogenesis are impaired in these contexts.

The study by Shiojima et al. (15) raises additional questions, which future studies will undoubtedly address: (a) Does increased injury account for all of the reduction in cardiac function in Akt 1 transgenic mice, or could additional mechanisms such as altered myocardial excitation-contraction coupling or altered myocardial energetics exist?; (b) Does the fibrosis observed represent increased cell death via necrosis or apoptosis, or are profibrotic signaling pathways activated? It is possible that if Akt-dependent pathways such as that resulting in VEGF production can be downregulated with chronic Akt expression; could this also be true of prosurvival/antiapoptotic pathways?; and (c) What is the mechanism responsible for the downregulation of TOR-mediated signaling to S6 kinase (S6K) and VEGF but not to myocyte growth that is seen in mice with chronic Akt activation? The results of S6K $\mathrm{KO}$ mouse studies suggest that S6K is not required for mediating pathological and physiological cardiac hypertrophy; however, rapamycin clearly blocks Akt-induced cardiac hypertrophy and leads to regression of pressure-overload hypertrophy (21, 22). Therefore, the additional mammalian TOR (mTOR) targets that mediate cardiac hypertrophy need to be identified.

\section{Chronic Akt1 overexpression and the heart's recovery from I/R injury}

Nagoshi and colleagues report that in contrast to mice with lower level and mosaic expression of a myristoylated Akt1 transgene (transgenic mouse line TG20), which their group previously reported to exhibit reduced infarct size following regional $\mathrm{I} / \mathrm{R}$ in vivo (11), a transgenic mouse line express- ing higher levels of Akt (transgenic mouse line TG564) failed to recover any function after global I/ $\mathrm{R}$ in an isolated heart model and developed larger myocardial infarctions (14). The basis for myocardial injury is likely increased necrosis and not increased apoptosis. The results of the Nagoshi et al. study suggest that downregulation of IRS-1/2mediated PI3K activation is an important contributor to the increased susceptibility to $\mathrm{I} / \mathrm{R}$ injury in this model. IRS- $1 / 2$ levels are reduced because of decreased expression of their respective genes as well as increased proteasomal degradation (Figure 1B). The I/R phenotype can be rescued by delivery of an adenovirus that harbors a constitutively active form of PI3K. These data support the notion of the presence of Akt-independent but PI3K-dependent cardioprotective signaling pathways that are lost when IRS-1-mediated signal transduction is impaired in the face of long-term Akt activation. This conclusion is also supported by the observations by Nagoshi et al. that IRS-1 is depleted to a much lesser extent in the transgenic mouse line TG20, which was previously reported to exhibit enhanced recovery from I/R injury in

\section{[A third study] in this issue of the $\mathrm{JCl}$. . now provides very strong evidence that Akt1 is the specific Akt isoform that mediates adaptive angiogenesis.}

vivo (11), and that transgenic mice that overexpress IGF-1 (which does not downregulate IRS-1 content despite an approximate 6-fold increase in Akt activation) have enhanced recovery from ischemic injury (14).

The major activation of IRS- $1 / 2$ signaling to PI3K in vivo occurs via tyrosine kinase receptors such as the insulin and IGF-1 receptors. Nagoshi et al. also show that in heart samples from humans with dilated cardiomyopathy, Akt activation is associ- 


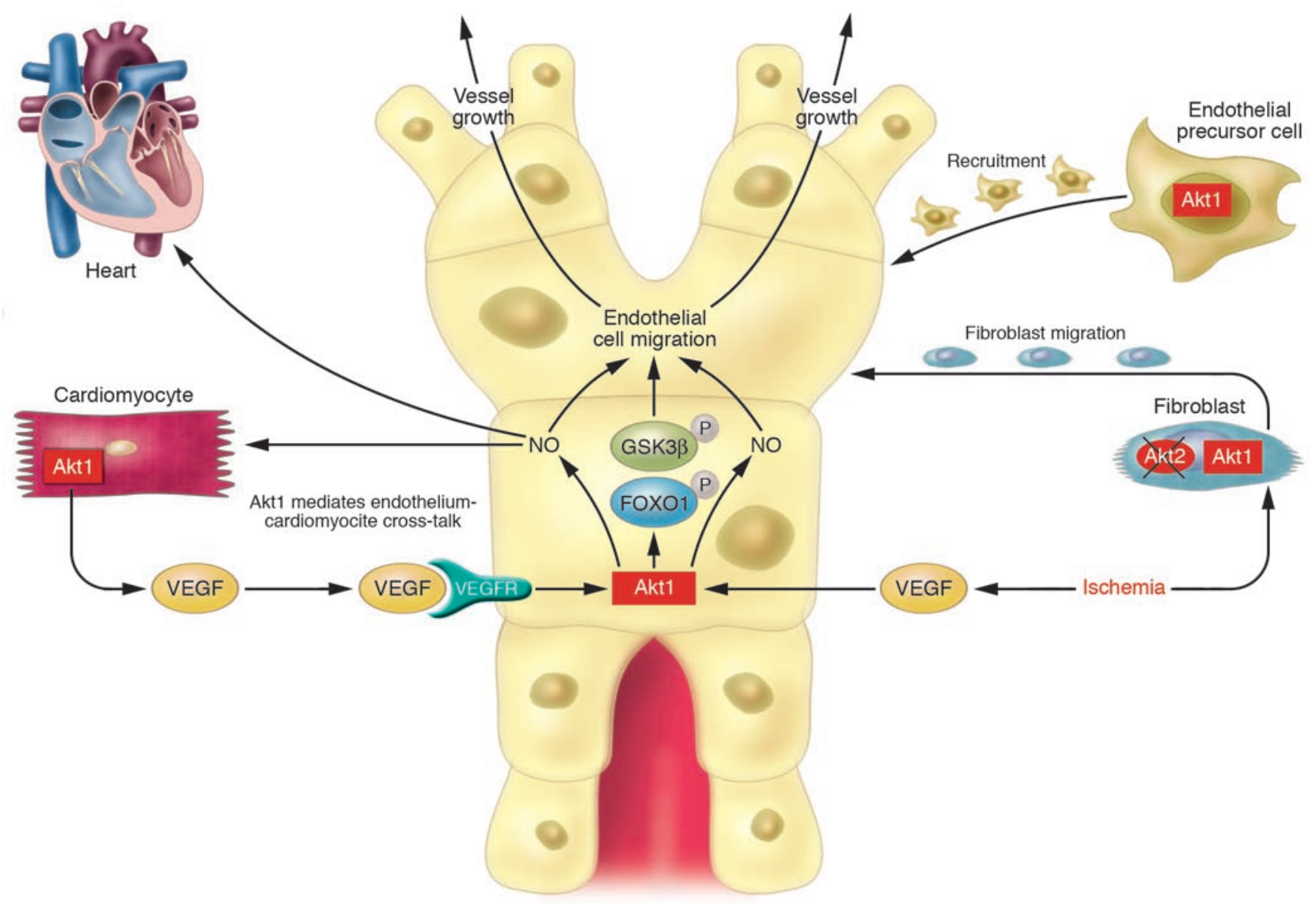

Figure 2

Akt1 promotes adaptive angiogenesis. In endothelial cells and VSMCs, Akt1 represents the predominant isoform. VEGF-mediated angiogenesis requires Akt1 activation. Angiogenesis is mediated in part by Akt1-dependent activation of eNOS, which leads to increased NO production. VEGF

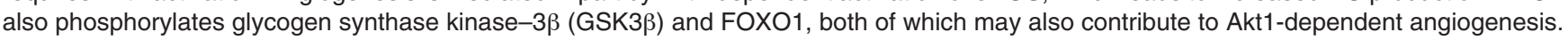
Akt1 is also required for the recruitment of bone marrow-derived endothelial precursor cells to sites of ischemia and for the migration of mature endothelial cells and fibroblasts to areas of active new vessel formation. Note that although Akt2 is expressed in fibroblasts, its expression is not required for promoting fibroblast migration. Akt1-mediated cross-talk between endothelium and cardiomyocyte involves the release of VEGF from cardiomyocytes, which may influence the vascular response to hypertrophy. It is also likely that Akt1-mediated release of NO from the vasculature will have important regulatory effects on the cardiomyocyte.

ated with reduced IRS-1 levels (14), which raises the intriguing possibility that loss of IRS-1-mediated PI3K signal transduction may contribute to the cardiac injury in these patients. Thus these results underscore the importance of signaling to IRS- $1 / 2$ via these growth factors/hormones in mitigating the myocardial response to ischemic injury. Results obtained from 3 other mouse models with impaired PI3K/Akt signaling also support the paradigm that growth factors signaling to PI3K are critical modulators of cardiac injury in response to stress: mice with cardiomyocyte-restricted $\mathrm{KO}$ of insulin receptors exhibit reduced cardiac performance and increased injury in response to pressure overload (23); mice with musclespecific KO of phosphoinositide-depen- dent kinase 1 that fail to phosphorylate Akt on Thr308 (although Ser473 is hyperphosphorylated) die prematurely from a dilated cardiomyopathy (24); and mice with a dominant-negative PI3K transgene do not develop exercise-induced hypertrophy, but develop LV dysfunction following pressureoverload hypertrophy (16). Taken together, these data indicate that proximal signaling inputs to PI3K and Akt (such as insulin) play important regulatory roles in the adaptation of the heart to diverse stresses such as ischemia and pressure-overload hypertrophy.

The current study by Nagoshi et al. (14) raises a number of questions and hypotheses that should be examined in future studies. First, what are the PI3K-dependent but Aktindependent pathways that are responsible for cardioprotection, and are these pathways activated by alternative signaling pathways such as $G$ protein-coupled receptors that may activate PI3K independently of IRS-1/2? It is unlikely that Akt-mediated protective pathways are not important, as shown by the impaired functional recovery in dominant-negative Akt transgenic or Akt1 $1^{-/-}$mice following I/R injury. It would therefore be interesting to determine the consequence of PI3K activation in Akt1-null hearts on their recovery from ischemic injury.

Second, Nagoshi and colleagues show that IRS-1 levels are increased in $A k t 1^{-/-}$ mice. Conversely, Akt 1 overexpression promotes proteasomal degradation of IRS-1 that is not mediated via the mTOR pathway (14). What, then, are the specific mecha- 
nisms that lead to IRS-1 degradation in this model? There is much evidence that serine phosphorylation of IRS-1 mediates its proteasomal degradation and that there are a variety of serine/threonine kinases that can mediate this process (25). It will therefore be important to determine whether Akt is directly responsible for the serine phosphorylation of IRS-1 or whether the responsibility lies in an Akt-dependent kinase.

Third, what are the mechanisms that lead to increased cell death in Akt transgenic mice following I/R? The report by Shiojima et al. (15) raises the intriguing possibility that decreased capillarity could limit capillary recruitment during reperfusion, which could contribute to increased injury. Another possibility is that changes in energetics that occur on the basis of chronic Akt activation may precipitate more rapid depletion of high energy phosphates or impair ATP regeneration upon reperfusion. In this regard, studies in E40K Akt transgenic mice suggest that 1 mechanism for increased contractility in these mice is increased activity of the sarcoplasmic endoplasmic reticulum $\mathrm{Ca}^{2+}$ ATPase, which would increase the rate of ATP utilization (26).

Finally, it would be of interest to determine the time course for IRS- 1 degradation in vivo using the inducible Akt model. A critical experiment would be to determine the extent of IRS-1/PI3K activation in the initial 2-week window during which the phenotype of these mice is completely reversible and determine whether recovery from I/R injury is normal or impaired following short-term Akt activation.

In summary, these 2 transgenic mouse models $(14,15)$ define important Aktdependent feedback mechanisms that lead to cardiac maladaptation. These include feedback inhibition of VEGF synthesis, which impairs angiogenesis, as well as inhibition of IRS- $1 / 2$ expression and IRS-mediated PI3K activation (Figure 1B). Akt has many more substrates within the cell. No doubt there will be a growing list of other signaling pathways that may be inhibited by long-term unregulated Akt signaling, such as the recently reported increase in insulin receptor threonine phosphorylation by Akt1-mediated pathways that leads to impaired insulin signaling (27). Stay tuned.

\section{Akt1 and adaptive angiogenesis}

A persuasive case for a role of the Akt 1 isoform in the regulation of postnatal adaptive angiogenesis and arteriogenesis (as opposed to developmental angiogenesis) is made in this issue of the JCI by Ackah and colleagues (18). In contrast to the expression seen in the heart and intact blood vessels, which express both Akt 1 and Akt2, there appears to be low-level expression of Akt2 in endothelial and vascular smooth

\section{Akt1-mediated cross-talk between endothelium and cadiomyocyte . . may influence the vascular response to hypertrophy.}

muscle cells. Thus in the vasculature, Akt 2 might be predominantly expressed in adventitial fibroblasts. Ackah et al. (18) demonstrate that Akt1, but not Akt2, mediates the angiogenic response to hind-limb ischemia and VEGF stimulation in vivo (Figure 2). Moreover, Akt1 is required for the homing of endothelial precursor cells to areas of ischemia in vivo. Akt 1 is also required for basal and agonist-mediated NO release from mouse lung endothelial cells (MLECs) and for the migration of MLECs (which express Akt1) and mouse lung fibroblasts (which express both Akt 1 and Akt2) in response to chemoattractants in vitro. In light of the evidence provided by Shiojima and colleagues showing that a coordinated balance between angiogenesis and cellular hypertrophy is essential for an adaptive response to physiological hypertrophy (15), the results of Ackah et al. (18) raise interesting questions regarding the potential role of Akt1-mediated angiogenesis in the failed induction of cardiac hypertrophy by exercise in mice expressing a dominant-negative PI3K transgene (16) or in the increased injury observed in cardiomyocyte-specific insulin receptor knockout (CIRKO) mice following pressure overload (23). Does impaired VEGF-mediated angiogenesis therefore retard physiological hypertrophy or promote injury in the hearts of mice with impaired PI3K/Akt signaling? This will be an important question to pursue in future studies.

In light of the novel specific roles for Akt 1 now defined in the vasculature and heart, it will be important to identify the specific downstream mediators of these effects. Akt continues to take center stage, and these recent studies have provided important new insights into the regulation of cardiac and vascular biology by this family of signaling molecules $(14,15$, $18)$. The studies have important implications for therapeutic strategies that may be based upon modulating Akt activity in the heart and vasculature in an effort to mitigate the consequences of heart failure and myocardial or other organ ischemia. In this installment of "Akt in the cardiovascular system," Akt 1 has clearly emerged as the star of Act 1 . We are sure that Act 2 will be equally interesting.

\section{Acknowledgments}

B.T. O'Neill is supported by a PhysicianScientist Training Award from the American Diabetes Association. E.D. Abel is supported by grants HL 73167, HL 70070, and HL 70525 from the National Institutes of Health and is an Established Investigator of the American Heart Association.

Address correspondence to: E. Dale Abel, Division of Endocrinology, Metabolism and Diabetes, Program in Human Molecular Biology and Genetics, 15 North 2030 East, Building 533 Room 3410B, Salt Lake City, Utah 84112, USA. Phone: (801) 5850727; Fax: (801) 585-0701; E-mail: dale. abel@hmbg.utah.edu.

1. Bellacosa, A., Testa, J.R., Moore, R., and Larue, L. 2004. A portrait of AKT kinases: human cancer and animal models depict a family with strong individualities [review]. Cancer Biol. Ther. 3:268-275.

2. Chen, W.S., et al. 2001. Growth retardation and increased apoptosis in mice with homozygous disruption of the Akt1 gene. Genes Dev. 15:2203-2208.

3. Cho, H., Thorvaldsen, J.L., Chu, Q., Feng, F., and Birnbaum, M.J. 2001. Akt1/PKBalpha is required for normal growth but dispensable for maintenance of glucose homeostasis in mice. J. Biol. Chem. 276:38349-38352.

4. Cho, H., et al. 2001. Insulin resistance and a diabetes mellitus-like syndrome in mice lacking the protein kinase Akt2 (PKB beta). Science. 292:1728-1731.

5. Easton, R.M., et al. 2005. Role for Akt3/protein kinase Bgamma in attainment of normal brain size. Mol. Cell. Biol. 25:1869-1878.

6. Peng, X.D., et al. 2003. Dwarfism, impaired skin development, skeletal muscle atrophy, delayed bone development, and impeded adipogenesis in mice lacking Akt1 and Akt2. Genes Dev. 17:1352-1365.

7. Oudit, G.Y., et al. 2004. The role of phosphoinositide-3 kinase and PTEN in cardiovascular physiology and disease [review]. J. Mol. Cell. Cardiol. 37:449-471.

8. Matsui, T., et al. 2001. Akt activation preserves cardiac function and prevents injury after transient cardiac ischemia in vivo. Circulation. 104:330-335.

9. Gnecchi, M., et al. 2005. Paracrine action accounts for marked protection of ischemic heart by Aktmodified mesenchymal stem cells. Nat. Med. 11:367-368.

10. Mangi, A.A., et al. 2003. Mesenchymal stem cells modified with Akt prevent remodeling and restore performance of infarcted hearts. Nat. Med. 9:1195-1201.

11. Matsui, T., et al. 2002. Phenotypic spectrum caused by transgenic overexpression of activated Akt in the heart. J. Biol. Chem. 277:22896-22901. 
12. Condorelli, G., et al. 2002. Akt induces enhanced myocardial contractility and cell size in vivo in transgenic mice. Proc. Natl. Acad. Sci. U. S. A. 99:12333-12338.

13. Shioi, T., et al. 2002. Akt/protein kinase B promotes organ growth in transgenic mice. Mol. Cell. Biol. 22:2799-2809.

14. Nagoshi, T., et al. 2005. PI3K rescues the detrimental effects of chronic Akt activation in the heart during ischemia/reperfusion injury. J. Clin. Invest. 115:2128-2138. doi:10.1172/JCI23073.

15. Shiojima, I., et al. 2005. Disruption of coordinated cardiac hypertrophy and angiogenesis contributes to the transition to heart failure. J. Clin. Invest. 115:2108-2118. doi:10.1172/JCI24682.

16. McMullen, J.R., et al. 2003. Phosphoinositide 3-kinase(p110alpha) plays a critical role for the induction of physiological, but not pathological, cardiac hypertrophy. Proc. Natl. Acad. Sci. U. S. A. 100:12355-12360.
17. McMullen, J.R., et al. 2003. The insulin-like growth factor 1 receptor induces physiological heart growth via the phosphoinositide 3-kinase(p110alpha) pathway. J. Biol. Chem. 279:4782-4793.

18. Ackah, E., et al. 2005. Akt1/protein kinase $B \alpha$ is critical for ischemic and VEGF-mediated angiogenesis. J. Clin. Invest. 115:2119-2127. doi:10.1172/ JCI24726.

19. Shioi, T., et al. 2000. The conserved phosphoinositide 3-kinase pathway determines heart size in mice. EMBO J. 19:2537-2548.

20. Yamashita, K., et al. 2001. Reperfusion-activated Akt kinase prevents apoptosis in transgenic mouse hearts overexpressing insulin-like growth factor-1. Circ. Res. 88:609-614

21. McMullen, J.R., et al. 2004. Deletion of ribosomal S6 kinases does not attenuate pathological, physiological, or insulin-like growth factor 1 receptorphosphoinositide 3-kinase-induced cardiac hypertrophy. Mol. Cell. Biol. 24:6231-6240.
22. McMullen, J.R., et al. 2004. Inhibition of mTOR signaling with rapamycin regresses established cardiac hypertrophy induced by pressure overload. Circulation. 109:3050-3055.

23. $\mathrm{Hu}, \mathrm{P}$., et al. 2003. Minimally invasive aortic banding in mice: effects of altered cardiomyocyte insulin signaling during pressure overload. Am. J. Physiol. Heart Circ. Physiol. 285:H1261-H1269.

24. Mora, A., et al. 2003. Deficiency of PDK1 in cardiac muscle results in heart failure and increased sensitivity to hypoxia. EMBO J. 22:4666-4676.

25. White, M.F. 2002. IRS proteins and the common path to diabetes. Am. J. Physiol. Endocrinol. Metab. 283:E413-E422.

26. Kim, Y.K., et al. 2003. Mechanism of enhanced cardiac function in mice with hypertrophy induced by overexpressed Akt. J. Biol. Chem. 278:47622-47628.

27. Morisco, C., et al. 2005. Akt mediates the cross-talk between beta-adrenergic and insulin receptors in neonatal cardiomyocytes. Circ. Res. 96:180-188.

\title{
A new direction for gene therapy: intrathymic T cell-specific lentiviral gene transfer
}

\author{
Ruth Seggewiss and Cynthia E. Dunbar
}

National Heart, Lung, and Blood Institute, NIH, Bethesda, Maryland, USA.

\begin{abstract}
Reports of neoplasia related to insertional activation of protooncogenes by retroviral vectors have raised serious safety concerns in the field of gene therapy. Modification of current approaches is urgently required to minimize the deleterious consequences of insertional mutagenesis. In this issue of the JCI, Adjali and colleagues report on their treatment of SCID mice lacking the 70-kDa protein tyrosine kinase, ZAP-70, with direct intrathymic injection of a ZAP-70-expressing T cell-specific lentiviral vector, which resulted in $T$ cell reconstitution (see the related article beginning on page 2287). Using lentiviral vectors and in situ gene transfer may represent a safer approach than using retroviral vectors for ex vivo gene transfer into HSCs, avoiding 3 factors potentially linked to leukemogenesis, namely HSC targets, ex vivo transduction and expansion, and standard Moloney leukemia virus-based retroviral vectors.
\end{abstract}

\section{SCID and its current standard of therapy}

SCID disorders are a family of genetically determined conditions characterized by a block in $\mathrm{T}$ cell development and function, variably associated with defects in other hematopoietic lineages. The genetic and phenotypic heterogeneity among these disorders is considerable. Affected children present with severe opportunistic infections due to a lack of functional mature $\mathrm{T}$ cells. The most serious forms of SCID are fatal in the first years of life (1). The only curative standard of therapy is an allogeneic bone

Conflict of interest: The authors have declared that no conflict of interest exists.

Citation for this article: J. Clin. Invest. 115:2064-2067 (2005). doi:10.1172/JCI26401. marrow transplant from an HLA-matched, related donor. If this is not possible, bone marrow transplantation from an HLAmatched, unrelated or a parental haploidentical donor is performed. The survival rate after HLA-matched transplantation is greater than $75 \%$ but falls markedly with the use of alternative donors, including haploidentical family donors and unrelated donors, and chronic disabling complications such as graft-versus-host disease are common. Some children reach full functional immunity following transplantation, but others fail to have their $\mathrm{B}$ cell function restored or can experience progressive loss of $\mathrm{T}$ cell immunity and even complete graft failure over time. These poor outcomes are particularly common when children are transplanted with non-HLA-matched donor cells, for instance from a parent or an unrelated donor $(2,3)$.

\section{Leukemia induction after successful oncoretroviral gene transfer in SCID-X1}

These unsatisfying outcomes, particularly with alternative donor transplantation, have stimulated intense interest in gene therapy approaches to SCID. The most common SCID subtype is SCID$\mathrm{X} 1$, an X-linked recessive disease characterized by a block in $\mathrm{T}$ and natural killer cell differentiation due to mutations in the common $\gamma$ chain of cytokine receptor complexes. Recently, complete immune reconstitution of young boys with SCID$\mathrm{X} 1$ by transduction of their $\mathrm{CD} 34^{+}$bone marrow cells with an oncoretroviral vector encoding the common $\gamma$ chain followed by reinfusion of transduced cells without conditioning therapy was reported (Figure 1A) $(4,5)$. These encouraging results represented the first unequivocal demonstration of the clinical efficacy of gene therapy. However, elation was short-lived, as 3 out of 11 enrolled children in this clinical trial have developed $\mathrm{T}$ cell leukemia linked to insertional mutagenesis, specifically, activation of the LIM domain only 2 (known as LMO2) transcription factor locus (6). Thus, at present the risks and potential benefits of gene therapy are being reconsidered. The 